\title{
ANÁLISE COMPARATIVA DO ALBEDO DE SUPERFÍCIE UTILIZANDO O ALGORITMO SEBAL PARA OS SISTEMAS SENSORES TERRA/MODIS E LANDSAT8/OLI NO MUNICÍPIO DE JATAÍ (GO)
}

\author{
Carlos Eduardo Damasceno $^{(\mathrm{a})}$, Alécio Perini Martins ${ }^{(\mathrm{b})}$ \\ (a)Laboratório de Geoinformação, Universidade Federal de Goiás/Regional Jataí, eduardo.geo@outlook.com.br. \\ (b)Laboratório de Geoinformação, Universidade Federal de Goiás/Regional Jataí, alecioperini@yahoo.com.br
}

\section{EIXO: GEOTECNOLOGIAS E MODELAGEM ESPACIAL EM GEOGRAFIA FÍSICA}

\begin{abstract}
Resumo:
O albedo de superfície é definido como a razão entre a radiação solar incidente e a radiação solar refletida. O modelo SEBAL é utilizado para calcular valores de evapotranspiração em superfície, sendo o cálculo do albedo a primeira etapa do modelo. A variação no albedo influencia diretamente nos padrões de evapotranspiração, atingindo o ciclo hidrológico e atuando na variação da precipitação local. O SEBAL foi aplicado para dois tipos de sistemas sensores (TERRA/MODIS e LANDSAT8/OLI) no intuito de fazer uma análise comparativa entre os resultados. Para aplicação do modelo, bem como para o processamento das imagens e geração dos mapas, foi utilizado o software ArcGis $10.1{ }^{\circledR}$ licenciado para o Laboratório de Geoinformação da Universidade Federal de Goiás/Regional Jataí. Foram utilizadas imagens do dia 07 de outubro de 2016 para o sistema LANDSAT8/OLI e do período entre 07 e 14 de outubro de 2016 para o sistema TERRA/MODIS.
\end{abstract}

Palavras chave: albedo de superfície, sensoriamento remoto, algoritmo SEBAL.

\section{Introdução}

O monitoramento de superfície, em especial de informações relacionadas ao balanço de energia vem, cada vez mais, sendo substituído por dados obtidos por satélites com auxílio de modelos matemáticos capazes de extrair informações das imagens geradas por estes sensores. Para realizar o imageamento da superfície, os sensores remotos trabalham com o balanço entre a principal fonte de energia que atinge a superfície terrestre - a radiação solar - e os padrões de reflectância dos diferentes objetos na superfície. O sensoriamento remoto, nada mais é do que a obtenção de informações sem o contato direto com o objeto estudado.

Para Ruhoff et al (2015, p. 4495) "um dos principais componentes do balanço de energia é o albedo, definido como a proporção do fluxo de energia refletida pela superfície em relação ao fluxo de energia incidente".

Os satélites/sensores utilizados para aquisição das imagens nesta pesquisa foram respectivamente: TERRA/MODIS, na semana do dia 07/10/2016 e Landsat8/OLI/TIRS, para imagem do dia 07/10/2016. 
Segundo Rosa,

O sensor MODIS (Moderate Resolution Imaging Spectroradiometer) é o principal instrumento a bordo do satélite Terra (EOS AM-1), um dos sistemas de observação da Terra da NASA. O MODIS realiza observações de toda a superfície terrestre a cada um ou dois dias, e adquire dados em 36 bandas espectrais que se situam entre 0,4 e $14,4 \mathrm{~mm}$ e se distribuem em diferentes grupos de resolução espacial. Estes dados contribuem para melhorar nossa compreensão da dinâmica global e os processos que ocorrem na terra, nos oceanos e na atmosfera mais baixa. O satélite se encontra em uma órbita circular, quase polar, hélio-sincrônica, a $705 \mathrm{~km}$ de altura. A hora solar de passada pelo nó descendente é às 10h30am (ROSA, 2009, p.125).

As bandas foram ordenadas de acordo com os comprimentos de onda. As bandas 1-19 e 26 formam o conjunto de bandas do espectro refletivo. As bandas 20-25, 27-36 formam o conjunto de bandas emissivas (ROSA, 2009).

Já o sistema Landsat, de acordo com Rosa (2009), foi desenvolvido pela NASA (National Aeronautics and Space Administration) com o objetivo de permitir a aquisição de dados espaciais, espectrais e temporais da superfície terrestre, de forma global, sinóptica e repetitiva.

O Landsat8 foi lançado no dia 11 de fevereiro de 2013 e possui dois sensores OLI e TIRS, que possuem onze bandas. O sensor OLI possui 8 bandas (1, 2, 3, 4, 5, 6, 7 e 9) multiespectrais com resolução geométrica de $30 \mathrm{~m}$ e uma banda (8) pancromática de $15 \mathrm{~m}$. O sensor TIRS possui duas bandas (10 e 11), com resolução de 100m, mas reamostrados em 30m para coincidir com as bandas multiespectrais do sensor OLI (USGS, 2016).

As estimativas de albedo foram executadas utilizando o algoritmo SEBAL (Surface Energy Balance Algorithms for Land), desenvolvido por Bastiaanssen (1995), a partir de princípios físicos envolvidos na reflexão, transmissão e absorção de energia pela superfície, necessitando de poucos dados coletados em campo e, portanto, permitindo o estudo de extensas áreas ou para regiões com poucos dados disponíveis ou de difícil acesso.

Nesse sentido, segundo Martins

O uso de modelos matemáticos, elaborados para extrair informações de imagens de satélite e dados coletados em superfície, mesmo que pontualmente e com distribuição espacial insuficiente, se mostram como alternativa interessante para realizar estudos e monitoramentos em áreas com grande extensão territorial, de difícil acesso e/ou com escassez de informações coletadas em estações ou postos de observação (MARTINS, 2015, p.18).

O município de Jataí localiza-se na microrregião Sudoeste de Goiás (Figura 1) e possui uma área de $7.174 \mathrm{~km}^{2}$, no reverso da Cuesta do Caiapó, no divisor entre as bacias dos rios Araguaia e Paraná. Sua rede hidrográfica pertence à bacia do Paraná, sendo constituído de afluentes da margem direita do Rio Paranaíba (JATAÍ, 2017), em sua maioria da bacia do Rio Claro. A diversidade de ambientes 
XVII Simpósio Brasileiro

de Geografia Fisica Aplicada

I Congresso Nacional

de Geografia Física

\section{OS DESAFIOS DA GEOGRAFIA FÍSICA NA FRONTEIRA DO CONHECIMENTO \\ Instituto de Geociências - Unicamp \\ Campinas - SP \\ 28 de Junho à 02 de Julho de 2017}

encontrada no município permite que a caracterização e análise da dinâmica destes ambientes possam ser realizadas com o uso de sensoriamento remoto e geoprocessamento. Os diferentes usos da terra identificados apresentam comportamentos distintos em relação ao albedo da superfície terrestre.
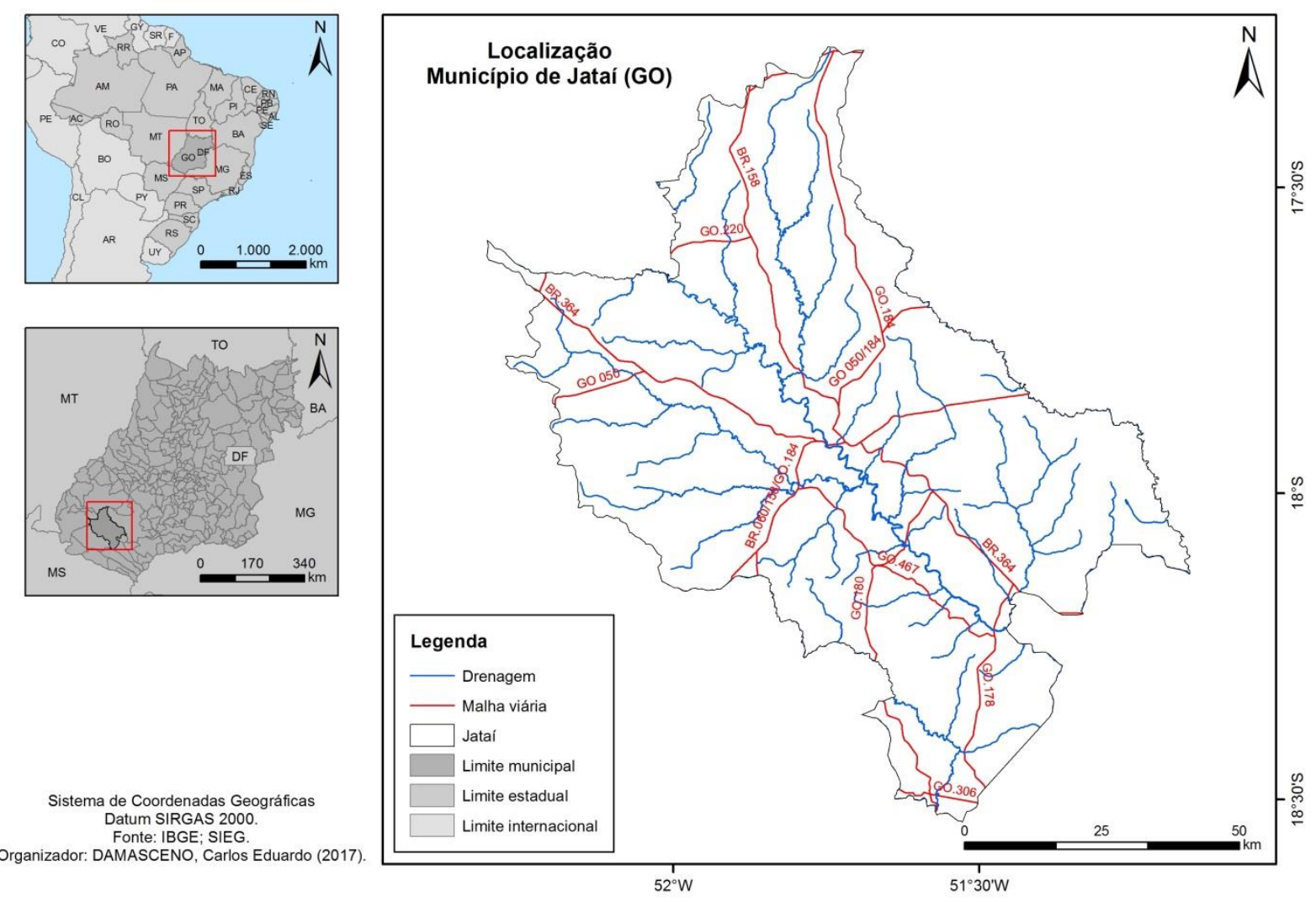

Figura 1 - Localização do município de Jataí (GO) Organizador: DAMASCENO, Carlos Eduardo, 2017

Os resultados aqui apresentados constituem parte de uma pesquisa de iniciação científica que visa avaliar a aplicação do modelo SEBAL para estimar valores de evapotranspiração na bacia do Rio Paranaíba. O objetivo central deste artigo é fazer uma análise comparativa entre os valores de albedo da superfície obtidos após o processamento de imagens dos satélites Terra e Landsat8 utilizando Sistemas de Informação Geográfica (SIG).

\section{Material e métodos}

Foram utilizados os produtos MOD09GA do sensor MODIS/TERRA (tile H13V10), abrangendo todo o território do município de Jataí, de uma composição de céu limpo entre os dias 07 e 14 de outubro de 2016 numa resolução espacial de 500m, onde foram georreferenciadas em Sistema de Coordenadas Geográficas, datum SIRGAS 2000, e realizado o recorte para a área de experimento (Figura 2). Cada pixel dos produtos MOD09GA contém a melhor observação L2G possível durante um período de oito dias, selecionado por cobertura de alta observação, ângulo de visão baixa, ausência de nuvens ou 
XVII Simpósio Brasileiro de Geografia Fisica Aplicada

I Congresso Nacional de Geografia Física

\section{OS DESAFIOS DA GEOGRAFIA FÍSICA NA FRONTEIRA DO CONHECIMENTO \\ Instituto de Geociências - Unicamp \\ Campinas - SP \\ 28 de Junho à 02 de Julho de 2017}

sombra de nuvens e carregamento de aerossol (USGS, 2017). Foi utilizado esse tipo de produto, pois a imagem do produto MOD09GQ do dia 07/10/2016 apresenta uma falha para a área de estudo.

Segundo Martins

\begin{abstract}
O albedo pode ser mensurado, em superfície, quando é possível realizar medições de radiação de ondas curtas incidente e refletida, o que limita muito a análise temporal e espacial desta variável em grandes superfícies. Nesse sentido, o sensoriamento remoto tem se mostrado útil para estimativa do albedo, como as disponibilizadas pelos produtos MOD43 e MYD43 dos satélites Terra e Aqua (sensor MODIS) ou pelo processamento das bandas de reflectância dos produtos MOD09 e MYD09. Assim gera-se um arquivo raster onde cada pixel traz informações diferentes sobre o albedo na área imageada, com variação ligada aos diferentes tipos de cobertura da superfície (MARTINS, 2015, p.33).
\end{abstract}

Foram utilizados também imagens OLI/Landsat8 do dia 07/10/2016, com resolução espacial de 30m, da órbita 223 e pontos 72 e 73, onde foram georreferenciadas em sistema de coordenadas geográficas, Datum SIRGAS 2000, e realizado o mosaico e recorte para a área de estudo (Figura 2).

Para o sistema sensor Landsat8/OLI foram utilizados os arquivos de metadados das imagens, disponibilizado junto com o download da imagem. O primeiro passo foi calcular a radiância espectral no topo da atmosfera para as bandas 2 a 7 (equação 1)(ALLEN, et al, 2002).

$$
L_{\lambda}=M_{L} Q_{c a l}+A_{L}
$$

onde $\mathrm{L}_{\lambda}$ corresponde a radiância espectral no topo da atmosfera, ML corresponde ao fator multiplicativo de reescalonamento para cada banda (encontrado nos metadados da imagem), $A_{L}$ corresponde ao fator aditivo de reescalonamento para cada banda (encontrado nos metadados da imagem) e Qcal corresponde ao número digital para cada pixel.

O segundo passo é calcular a reflectância planetária no topo da atmosfera $(\rho \lambda)$ dada pela equação 2 (ALLEN, et al, 2002).

$$
\rho_{\lambda}=\frac{\pi L_{\lambda} d^{2}}{E S U N_{\lambda} \cos \theta_{s z}}
$$

onde $\pi$ corresponde a constante $3,1416, \mathrm{~d}$ corresponde a distância astronômica entre Terra-Sol (encontrado nos metadados da imagem), $\mathrm{ESUN}_{\lambda}$ corresponde a irradiância solar média no topo da atmosfera e $\theta$ é a subtração entre $90^{\circ}$ e o azimute solar (encontrado nos metadados da imagem). Os valores do $\mathrm{ESUN}_{\lambda}$ foram calculados por Ruhoof et al. (2015).

O terceiro passo é calcular o albedo no topo da atmosfera ( $\alpha$ toa) pela equação 3, utilizando os valores determinados por Ruhoof et al. (2015) para o Landsat 8.

$$
\alpha_{\text {toa }}=0,300 \rho_{2}+0,276 \rho_{3}+0,233 \rho_{4}+0,143 \rho_{5}+0,035 \rho_{6}+0,012 \rho_{7}
$$

A quarta etapa consiste em calcular a transmissividade ( $\tau \mathrm{sw})$, dada pela equação 4 (ALLEN, et al, 2002). 
XVII Simpósio Brasileiro

de Geografia Física Aplicada

I Congresso Nacional

de Geografia Física

\section{OS DESAFIOS DA GEOGRAFIA FÍSICA NA FRONTEIRA DO CONHECIMENTO \\ Instituto de Geociências - Unicamp \\ Campinas - SP \\ 28 de Junho à 02 de Julho de 2017}

$$
\tau_{s w}=0.75+2 * 10^{-5} * z
$$

onde z é a altitude da área de estudo. Neste caso foi utilizada a altitude média para o município de Jataí, no valor de $750 \mathrm{~m}$.

Por fim, calcula-se o albedo da superfície ( $\alpha$ ), conforme a equação 5 (ALLEN, et al, 2002).

$$
\alpha=\frac{\alpha_{\text {toa }}-\alpha_{\text {path }} \text { radiance }_{\text {s }}}{\tau_{s w}^{2}}
$$

onde $\alpha_{\text {path_radiance }}$ é uma constante recomendada pelo SEBAL no valor de 0,03 , baseada em Bastiaanssen (2000).

Os sistemas sensores Aqua/MODIS e Terra/MODIS, de acordo com Martins (2005) apresentam processamento de correção atmosférica e informações de radiância e refletividade. Dessa forma, utilizou-se um procedimento descrito por Nicácio (2008) e Andrade et al (2010), conforme equação 6. Antes de executar a equação é necessário multiplicar cada banda do MOD09 por um valor de correção de 0,0001 .

$$
\alpha=0,160 \rho_{1}+0,291 \rho_{2}+0,243 \rho_{3}+0,116 \rho_{4}+0,112 \rho_{5}+0,081 \rho_{7}-0,0015
$$

onde $\rho_{2}, \rho_{3} \ldots \rho_{7}$ correspondem às bandas de reflectância do produto MOD09A1.

Para a aplicação dos cálculos de albedo, bem como o processamento das imagens e a criação dos mapas temáticos, foi utilizado o software ArcGis $10.1{ }^{\circledR}$ licenciado para o Laboratório de Geoinformação da Universidade Federal de Goiás/Regional Jataí, especialmente a ferramenta "Raster Calculator".

Após a realização das etapas acima foi feita uma média dos valores do albedo por pixel para comparação dos resultados considerando cada categoria de uso.

\section{Resultados}

\section{Uso da terra e cobertura vegetal}

De acordo com a tabela I observa-se que as áreas produtivas (agricultura e pecuária) ocupam a maior parte do território, totalizando 61,97\%, isso deve-se segundo Martins aos

Crescentes investimentos que transformaram, gradativamente, a paisagem: áreas de Cerrado e mata perdem espaço para novas pastagens; áreas de cerrado e antigas pastagens, gradativamente, são abertas para o plantio de soja, milho e, recentemente, cana-de-açúcar. Os municípios de Jataí, Rio Verde e Chapadão do Céu passam a figurar entre os maiores produtores de grãos do Estado de Goiás e do Brasil (MARTINS, et al, 2016, p. 12).

Os $21,70 \%$ classificados como Mata/Cerradão, são referentes a matas ciliares e de galeria, valor justificado por conta da rede hidrográfica do município (Figuras 2 e 3 ). 
XVII Simpósio Brasileiro de Geografia Fisica Aplicada

I Congresso Nacional de Geografia Física

\section{OS DESAFIOS DA GEOGRAFIA FÍSICA NA FRONTEIRA DO CONHECIMENTO \\ Instituto de Geociências - Unicamp \\ Campinas - SP \\ 28 de Junho à 02 de Julho de 2017}

Tabela I - Área $\left(\mathrm{km}^{2}\right)$ e porcentagem (\%) do uso da terra e cobertura vegetal para o município de Jataí-GO (2015)

\begin{tabular}{|c|c|c|}
\hline Uso & Área $\left.\mathbf{( k m}^{\mathbf{2}}\right)$ & Porcentagem \\
\hline Agricultura & 2552,66 & $35,60 \%$ \\
\hline Água & 10,5 & $0,15 \%$ \\
\hline Área urbana & 23,24 & $0,32 \%$ \\
\hline Cerrado & 974 & $13,58 \%$ \\
\hline Mata/Cerradão & 1556 & $21,70 \%$ \\
\hline Pastagem & 1891 & $26,37 \%$ \\
\hline Silvicultura & 47 & $0,66 \%$ \\
\hline Solo descoberto & 115,6 & $1,61 \%$ \\
\hline
\end{tabular}
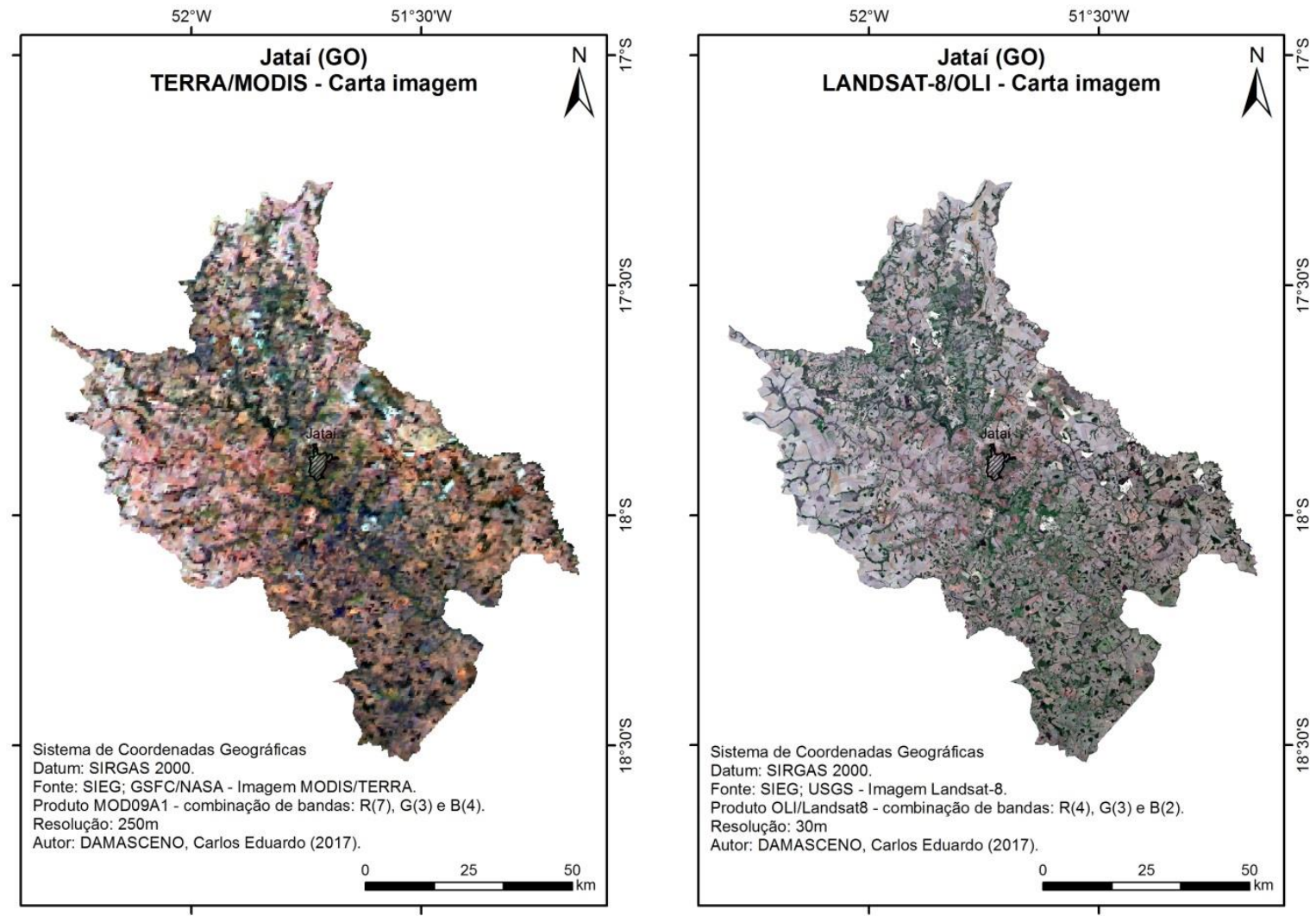

Figura 2 - A) Imagem TERRA/MODIS, produto MOD09GA, 2016 - R(7) G(3) B(4)

B) Imagem Landasat8, sensor OLI, 2016 - R(4) G(3) B(2)

Autor: DAMASCENO, Carlos Eduardo, 2017 


\section{OS DESAFIOS DA GEOGRAFIA FÍSICA NA FRONTEIRA DO CONHECIMENTO Instituto de Geociências - Unicamp Campinas - SP 28 de Junho à 02 de Julho de 2017}
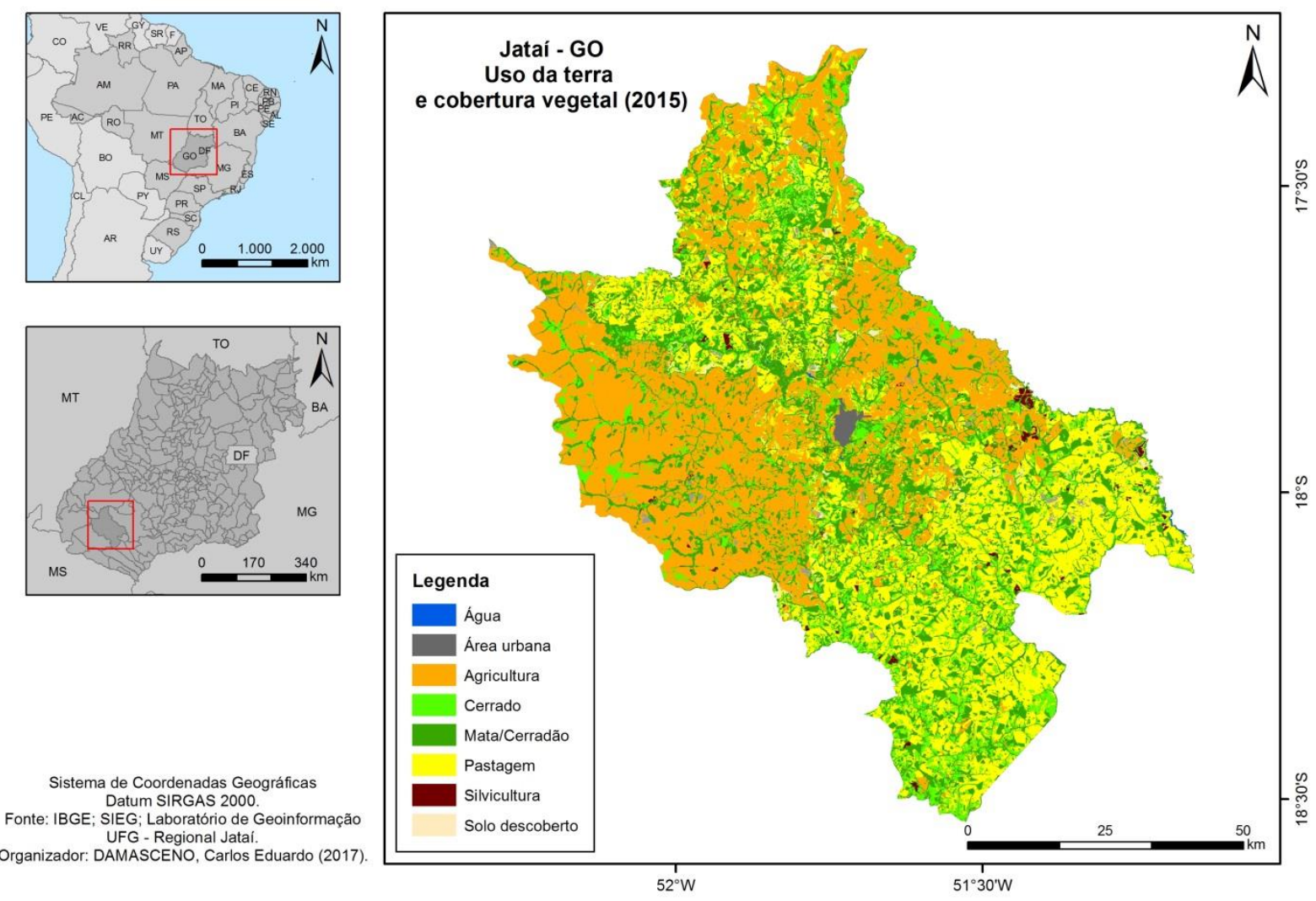

Figura 3 - Imagem Landsat/8, 2015

Organizador: DAMASCENO, Carlos Eduardo, 2017

\section{Albedo}

Foram feitas as análises a partir da média do valor do albedo para cada pixel das imagens de cada sensor (Tabela II). O resultado mostra-se satisfatório para ambos os sensores e a diferença dos resultados deve-se a resolução espacial dos sensores, MODIS apresentando uma resolução espacial de 500m e o LANDSAT apresentando resolução espacial de 30m (Figura 5).

Tabela /l - Valores médios de albedo por pixel dos sensores (LANDSAT/OLI e TERRA/MODIS), considerando cada categoria de uso (2015)

\begin{tabular}{|c|c|c|}
\hline \multirow{2}{*}{ Uso } & \multicolumn{2}{|c|}{ Sensor } \\
\cline { 2 - 3 } & OLI & MODIS \\
\hline Agricultura & 0,18 & 0,19 \\
\hline Cerrado & 0,13 & 0,15 \\
\hline Água & 0,06 & 0,10 \\
\hline Área urbana & 0,19 & 0,17 \\
\hline Mata/Cerradão & 0,12 & 0,17 \\
\hline Pastagem & 0,20 & 0,20 \\
\hline Silvicultura & 0,12 & 0,16 \\
\hline Solo descoberto & 0,24 & 0,22 \\
\hline
\end{tabular}



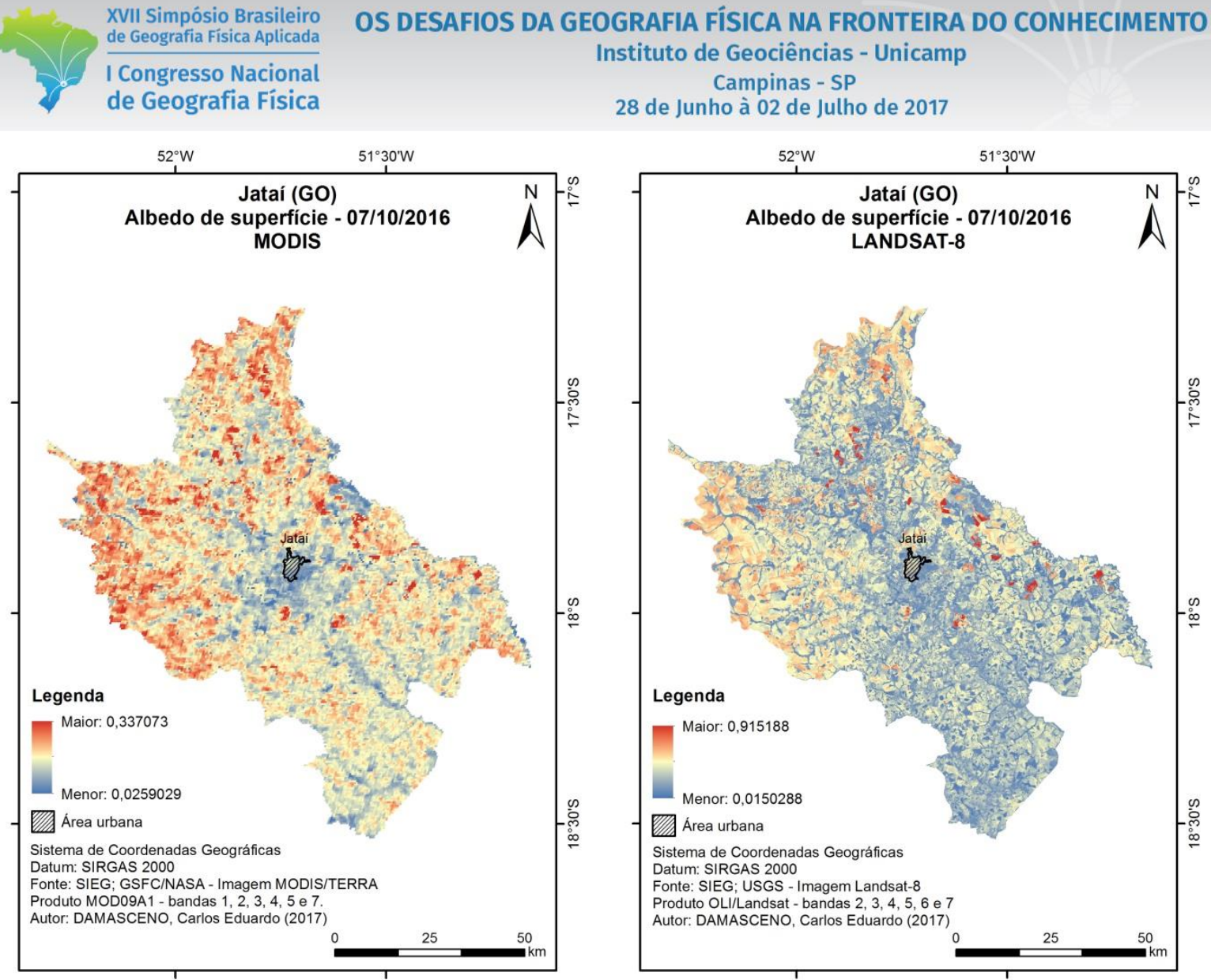

Figura 4 - Distribuição espacial dos valores de albedo para o município de Jataí-GO

Os valores mínimos de albedo foram apresentados em corpos d'água, com média de 0,06 (LANDSAT/OLI) e 0,10 (TERRA/MODIS), enquanto os maiores valores foram observados em área de solo descoberto, com média de 0,24 (LANDSAT/OLI) e 0,22 (TERRA/MODIS).

Vale ressaltar que na figura 5 o maior valor de albedo encontrado para o sistema sensor Landsat8/OLI foi de 0,91 , em áreas construídas, valores não identificados no processamento da imagem TERRA/MODIS devido à baixa resolução espacial. Segundo Martins (2015), alterações na cobertura do solo, podem provocar um aumento nas taxas de albedo e, consequentemente, interferir negativamente nos valores de evapotranspiração.

Para analisar os resultados, foi utilizada uma comparação com os resultados de Mendonça et al (2010), após cálculo do albedo de superfície utilizando o sensor MODIS, identificou valores de 0,14 a 0,18 para áreas de cana-de-açúcar em diferentes períodos do ano, assim como os encontrados nos resultados do processamento para o município de Jataí. Ming e Liu (2010) utilizando o sensor AVHRR/NOAA, encontraram valores de 0,10 até 0,15 para áreas de vegetação intensa, semelhantes aos encontrados pelo sensor OLI e com apenas 0,01 de diferença para o sensor MODIS. Segundo os mesmos autores, áreas de vegetação rasteira apresentam albedos entre 0,15 e 0,25, como nos resultados aqui encontrados. 


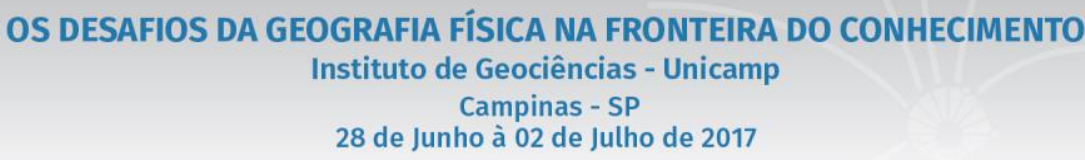

As maiores diferenças de albedo registradas entre os sensores foram encontradas nas áreas de água e mata, fato que se deve à resolução espacial de cada sensor (figura 5). O sensor OLI/LANDSAT tem resolução de 30m, já o sensor MODIS/TERRA apresenta resolução de 500m, ou seja, o nível de detalhamento do sensor OLI/LANDSAT é maior que o do MODIS/TERRA. Por outro lado, as imagens do sistema sensor MODIS/TERRA abrangem uma área maior, assim facilitando o trabalho para áreas de grande dimensão, não necessitando de mosaicos. Quanto ao mosaico, cada órbita do Landsat é captada em dias diferentes, com período de revisita de 16 dias, o que pode alterar o valor dos pixels quando se trabalha com áreas mais extensas, o que não ocorre com o sistema sensor TERRA/MODIS, que gera imagens diárias.

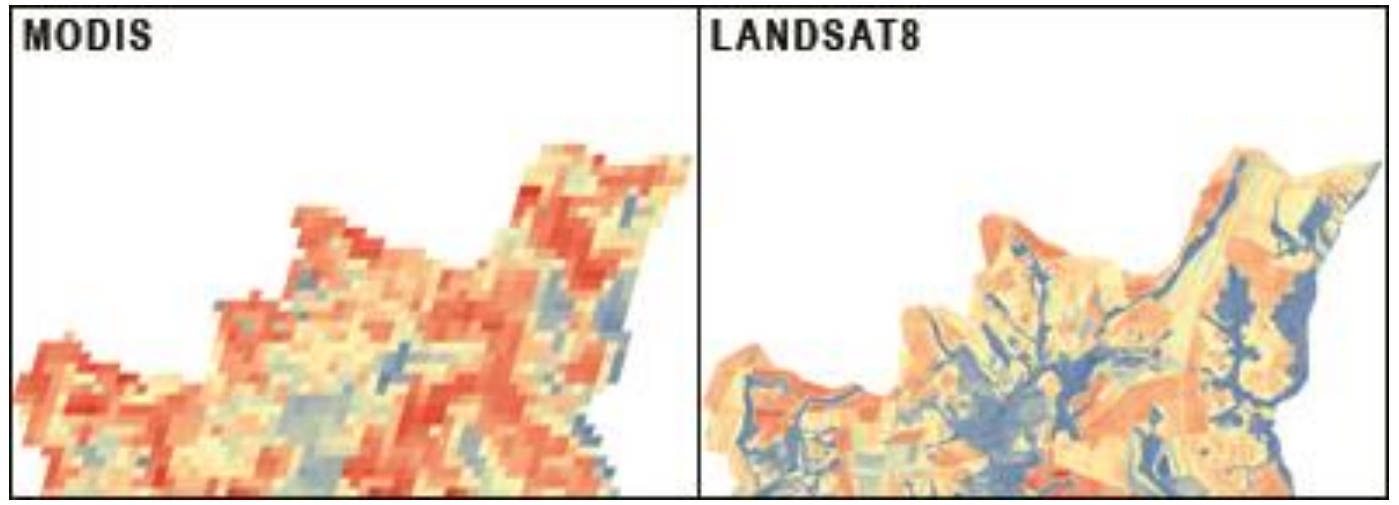

Figura 5 - Diferença de resolução espacial entre os sistemas sensores TERRA/MODIS e LANDSAT8/OLI utilizando os valores de albedo

\section{Considerações finais}

Proposta com intuito de fazer uma análise comparativa entre os sistemas sensores TERRA/MODIS e LANDSAT/OLI, utilizando sensoriamento remoto e ferramentas de geoprocessamento. Conclui-se que os resultados do albedo foram satisfatórios para ambos os sistemas sensores em comparação com valores encontrados nas referências pesquisadas. Porém, é valido ressaltar que para áreas pequenas e que exigem maior nível de detalhamento, é melhor utilizar o sistema sensor LANDSAT/OLI. Para regiões mais extensas, o sistema sensor TERRA/MODIS seria ideal, pois cobre uma área maior que a do sistema sensor LANDSAT/OLI.

\section{Agradecimento}

À agencia financiadora CNPq - PIBIC, pelo apoio financeiro.

\section{Referências bibliográficas}


ALLEN, R. G.; TASUMI, M.; TREZZA, R.; WATERS, R.; BASTIAANSSEN, W. G. M. Surface Energy

Balance Algorithm for Land (SEBAL)-Advanced Trainig and User's Manual, University of Idaho: Kimberly, EUA, p. 98, 2002.

ANDRADE, R. G. et al. Estimativa do albedo e do saldo de radiação à superfície utilizando imagem MODIS/AQUA na bacia do Rio Ji-Paraná/RO. Campinas, SP: Embrapa Monitoramento por Satélite, 2010. $24 \mathrm{p}$.

JATAÍ. Prefeitura de Jataí-GO. Disponível em < http://www.jatai.go.gov.br>. Acesso em 09 de fev. 2017.

MARTINS, A. P. Uso de dados do sensor Modis/Aqua e do algoritmo Sebal para estimativa da evapotranspiração real na bacia do Rio Paranaíba / Alécio Perini Martins - 2015.

MARTINS, A. P.; IRACI, S.; SOUSA, M. S.; PEIXINHO, D. M. Uso da terra e cobertura vegetal de 1985 a 2015 no Sudoeste de Goiás e relações com o meio Físico. In: PEIXINHO, D. M. Reconfiguração do cerrado: uso, conflitos e impactos ambientais / Dimas Moraes Peixinho; Marluce Silva Sousa (Org.). - Goiânia: 268 p. : il.

MENDONÇA, J. C. et al. Análise do albedo à superfície a partir de produtos MODIS. In: CBMET, CONGRESSO BRASILEIRO DE METEOROLOGIA, 16, 2010, Belém/PA. Anais do XVI Congresso Brasileiro de Meteorologia. Belém, 2010. p.1-5.

MODIS. Moderate Resolution Imaging Spectroradiometer. Disponível em <https://modis.gsfc.nasa.gov>. Acesso em 09 fev. 2017.

NICÁCIO, R. M. Evapotranspiração real e umidade do solo usando dados de sensores orbitais e a metodologia SEBAL na bacia do Rio São Francisco (Rio de Janeiro). 2008. 337f. Tese (Doutorado em Engenharia Civil) - Programa de Pós-Graduação em Engenharia Civil, Universidade Federal do Rio de Janeiro, 2008.

ROSA, R. Introdução ao sensoriamento remoto / Roberto Rosa. - 7. Ed. Uberlândia: EDUFU, 2009.

RUHOOF, A. L.; SILVA NOVO, B. B.; ROCHA, H. R. Determinação da irradiância solar no topo da atmosfera para cálculo do albedo e balanço de energia a partir de imagens LANDSAT 8 OLI. Anais. VVII Simpósio Brasileiro de Sensoriamento Remoto - SBSR, João Pessoa-PB, p. 4495-4502, 2015.

USGS. United States Geological Survey. Disponível em $<$ https://earthexplorer.usgs.gov>. Acesso em 09 fev. 2017. 